\title{
Editorial
}

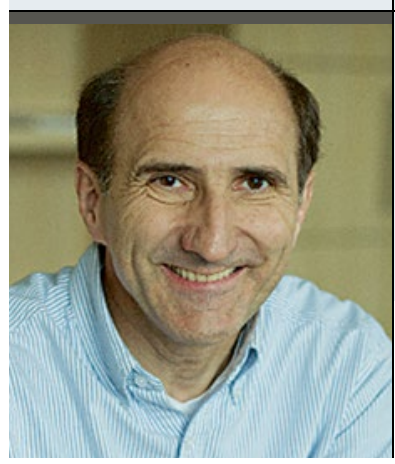

"Leider hat die neuere Literatur nur sehr begrenzt Eingang in die

neue S3-Leitlinie gefunden, wofür sicherlich auch die langen

Vorlaufzeiten verantwortlich sind."

Prof. Dr. med. Ralf Ihl

Maria-Hilf-Krankenhaus, Klinik für Gerontopsychiatrie und -psychotherapie, Krefeld

\section{S3-Leitlinie Demenz - Fakten, Einschätzungen und Hoffnungen}

A uch wenn sie es noch nicht auf die AWMFHomepage geschafft hat: Die neue S3-Leitlinie Demenzen liegt vor. So richtig viel Neues hat sich seit dem Beginn des Leitlinienprozesses zur Demenz 1994 allerdings nicht ergeben. Die damals empfohlenen Antidementiva sind die gleichen wie heute. Ernüchternd nach mehr als 20 Jahren Demenzforschung.

\section{Marker, Symptome ... und viele Hypothesen}

Über die Ursachen fehlenden Erfolgs wird derzeit weltweit gerätselt. Cholinerge und Amyloidhypothese wurden breit und umfassend untersucht, doch bahnbrechende Erfolge blieben aus. Es sei daran erinnert, dass Acetylcholin nur einer von vielen bei Demenz veränderten Botenstoffen ist, auch wenn mit seiner Bedeutung für die Informationsverarbeitungsprozesse ein plausibles Modell dahintersteht. Mit Verblüffung wurde festgestellt, dass wir in der Lage sind, das Amyloid aus dem Gehirn zu schaffen, die Krankheit aber bleibt. So richtig viel Glück hatten wir auch mit amyloidbezogenen Biomarkern nicht, Sensitivität und Spezifität bleiben weit entfernt von echtem klinischen Nutzen. Selbst das Gewebe von Auguste Deter, der ersten von Alois Alzheimer diagnostizierten Patientin, weigerte sich, den Biomarker Apolipoprotein $\varepsilon 4$ preiszugeben - es war homozygot für das Allel $\varepsilon 3$. Hier stellt sich auch die Frage, inwieweit Erkrankte die Konstellation von Markern und Symptomen der Ersterkrankten auch aufweisen müssen, um als an „Alzheimer-Demenz“ erkrankt zu gelten. Eine Vielzahl von Arbeiten beschäftigt sich seither mit Argumenten, warum die alten Hypothesen doch stimmen. Eine interessante, gute und sehr lang bekannte Erklärung, warum sich alles nicht einer stimmigen Hypothese unterwirft, ist der Hinweis auf eine mögliche Ursachenheterogenität. Dafür gibt es viele Belege.
Das positive an dem derzeitigen „Biomarker-Hy-

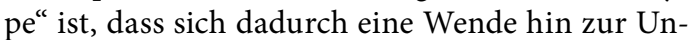
tersuchung homogenerer Gruppen vollzieht. Möglichkeiten dazu gab es allerdings schon weit vor Amyloid- und Tau-Messungen. Klinik, Testpsychologie und EEG boten sich an, um nur einige zu nennen. Genutzt wurden sie wenig. Auch die Phase zwischen gesund und krank beschäftigt die Forscher weiter. Diverse Definitionen zu Vorstadien der Demenz wurden in die Welt gesetzt (MCI etc.), nachvollziehbare Definitionen fehlen. Barry Reisbergs klärendes Werk bleibt in der Leitlinie unerwähnt. Was folgt, ist eine Heterogenität der Studiengruppen, die zu keinen oder arbiträren Ergebnissen führt. So entsteht eine schwierige Ausgangsbasis für weiterführende Erkenntnisse. Etwas Hoffnung keimt auf, wenn man sieht, dass andere Hypothesen, wie die Entzündungshypothese, wieder stärker in den Vordergrund rücken. Die amerikanische Alzheimer-Gesellschaft hat sie zum aktuellen Forschungsschwerpunkt erklärt und fördert entsprechende Projekte mit beachtlichen Mitteln.

\section{Was kommt außer Medikation infrage?}

Doch was tun, bis Medikamente mehr als moderate Effekte bei einzelnen Patienten zeigen? Hier tut sich die Leitlinie mittlerweile weniger schwer. Sie nennt eine Vielzahl von psychosozialen Interventionen, zu denen auch bessere Belege vorgelegt worden sind. Die methodischen Anforderungen an Studien im psychosozialen Bereich sind nicht durchgängig deckungsgleich mit den Anforderungen an Medikamentenstudien. Untersuchungen im Umfeld erfordern ein anderes Vorgehen. Die Risiken von nicht erkannten Wirkungen und falsch positiven Erkenntnissen sind hoch. Die Methodik dazu befindet sich noch in einem Entwicklungsstadium. Immerhin hat sich bereits auch das IQWIG der Problematik angenommen. Manches Dogma, wie das 


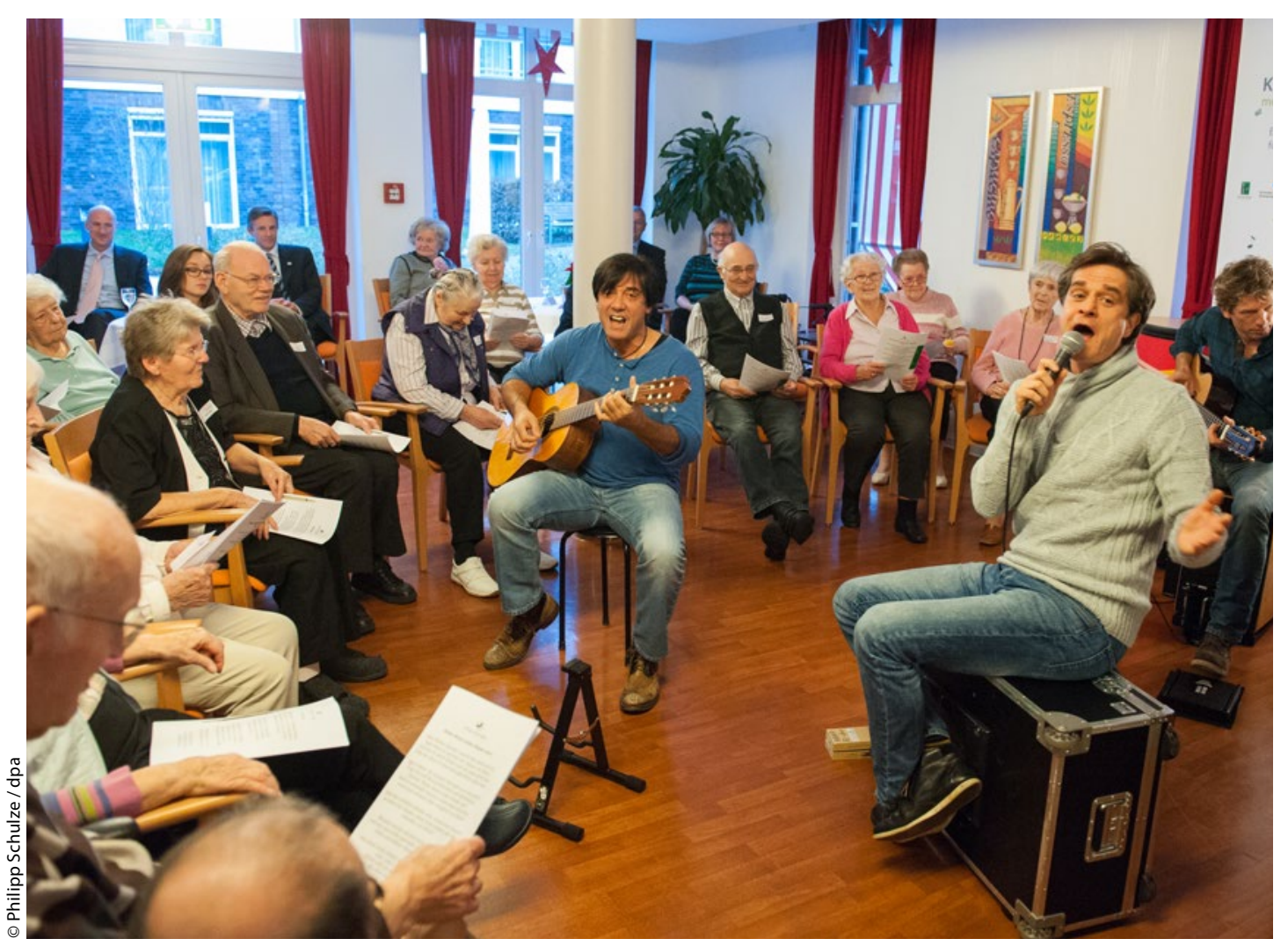

In der neuen Leitlinie heißt es: Kognitive Stimulation sollte empfohlen/angeboten werden. Auch Verfahren wie Musiktherapie können empfohlen werden. Beispiel: Im Rahmen des Projekts „Klang und Leben“ versuchen Musiker und ein Demenzcoach bei Senioren alte Erinnerungen mit Gesang und Musik zu wecken.

der Placebogruppe wurde hinterfragt. Das war spätestens nach dem Hinweis auf fehlende placebokontrollierte Studien zur Schutzwirkung von Fallschirmen bei Sprüngen aus großer Höhe notwendig geworden. Es wäre ein Armutszeugnis der Wissenschaft, wenn sie hierfür keine alternativen Lösungen entwickeln könnte. Gerade bei komplexen Untersuchungssettings wie bei der umfassenden ambulanten Betreuung von Menschen mit Demenz wäre eine Weiterentwicklung kaum zu erwarten. In der Leitlinie finden Netzwerke bisher wohl nicht zuletzt deswegen keine Erwähnung.

Bei Unruhe, Aggression und anderen den Umgang erschwerenden Verhaltensweisen sind medikamentöse Empfehlungen weiterhin nur wenig durch Daten begründet. Wichtig, dass Risiken wie eine erhöhte Mortalität unter Neuroleptika Eingang in die Leitlinie gefunden haben. Dass Neuroleptika mit Abstrichen aber doch empfohlen werden, bestätigt dann wieder die schon vorher bestehende Unsicherheit. Die neuere Literatur hat leider nur sehr begrenzt Eingang gefunden, wofür sicherlich auch die langen Vorlaufzeiten von Leitlinien verantwortlich sind.
Nun scheint es zwar gelungen, Neurologie, Psychiatrie und Hausärzte zusammenzubringen, das schützt aber nicht vor Leitlinien mit großem Überschneidungspotenzial wie etwa von der Nuklearmedizin zur Amyloidbildgebung und von der Neurologie zu Gedächtnisstörungen. Ob die Flut von mehr als 100.000 wissenschaftlichen Artikeln zur Demenz durch längere und mehr Leitlinien von vielen Nationen und Gruppierungen leichter beherrschbar wird, bleibt zu bezweifeln (siehe auch DNP 4/2014). Bis mehr Fakten vorhanden sind beziehungsweise wahrgenommen und umgesetzt werden, verlassen wir uns auf Einschätzungen und Hoffnungen.

Die DNP-Redaktion hat die zentralen Aussagen der Demenzleitlinie kurz und prägnant zusammengefasst. Aus der Leitliniengruppe heraus werden Aussagen zu neuropsychiatrischen Störungen auf den Punkt gebracht. Wem die Zeit zum ausführlichen Literaturstudium fehlt, erhält in dieser Ausgabe einen raschen Überblick.

Viel Freude beim Lesen wünscht Ihnen 